\title{
H.E.S.S. observations of the flaring gravitationally lensed galaxy PKS 1830-211
}

H.E.S.S. Collaboration, H. Abdalla, ${ }^{1}$ F. Aharonian, ${ }^{2,3,4}$ F. Ait Benkhali, ${ }^{2}$

E. O. Angüner, ${ }^{5}$ M. Arakawa,${ }^{6}$ C. Arcaro, ${ }^{1}$ C. Armand,${ }^{7}$ M. Arrieta,${ }^{8}$ M. Backes,${ }^{1,9}$

M. Barnard, ${ }^{1}$ Y. Becherini, ${ }^{10}$ J. Becker Tjus,${ }^{11}$ D. Berge,${ }^{12} \mathrm{~K}$. Bernlöhr, ${ }^{2}$

R. Blackwell, ${ }^{13}$ M. Böttcher, ${ }^{1}$ C. Boisson, ${ }^{8} \mathrm{~J}$. Bolmont, ${ }^{14} \mathrm{~S}$. Bonnefoy, ${ }^{12 \star} \mathrm{P}$. Bordas, ${ }^{2}$ J. Bregeon, ${ }^{15}$ F. Brun, ${ }^{16}$ P. Brun, ${ }^{16 \star}$ M. Bryan, ${ }^{17}$ M. Büchele, ${ }^{18}$ T. Bulik, ${ }^{19}$ T. Bylund, ${ }^{10}$ M. Capasso, ${ }^{20}$ S. Caroff, ${ }^{21}$ A. Carosi, ${ }^{7}$ S. Casanova,,${ }^{2,22}$ M. Cerruti,,${ }^{14} \dagger$ N. Chakraborty, ${ }^{2}$ T. Chand, ${ }^{1}$ S. Chandra, ${ }^{1}$ R. C. G. Chaves,${ }^{15} \ddagger$ A. Chen,${ }^{23}$ S. Colafrancesco,${ }^{23} \S$

B. Condon, ${ }^{24}$ I. D. Davids, ${ }^{9}$ C. Deil,${ }^{2}$ J. Devin, ${ }^{15}$ P. deWilt, ${ }^{13}$ L. Dirson, ${ }^{25}$

A. Djannati-Ataï, ${ }^{26}$ A. Dmytriiev, ${ }^{8}$ A. Donath,${ }^{2}$ V. Doroshenko, ${ }^{20}$

L. O'C. Drury, ${ }^{3}$ J. Dyks, ${ }^{27}$ K. Egberts, ${ }^{28}$ G. Emery, ${ }^{14}$ J.-P. Ernenwein, ${ }^{5}$ S. Eschbach, ${ }^{18}$

S. Fegan, ${ }^{21}$ A. Fiasson, ${ }^{7}$ G. Fontaine,${ }^{21}$ S. Funk,,${ }^{18}$ M. Füßling, ${ }^{12}$ S. Gabici, ${ }^{26}$

Y. A. Gallant, ${ }^{15}$ F. Gaté, ${ }^{7}$ G. Giavitto, ${ }^{12}$ D. Glawion, ${ }^{29}$ J. F. Glicenstein ${ }^{\odot},{ }^{16 \star}$

D. Gottschall, ${ }^{20}$ M.-H. Grondin, ${ }^{24}$ J. Hahn, ${ }^{2}$ M. Haupt, ${ }^{12}$ G. Heinzelmann, ${ }^{25}$ G. Henri, ${ }^{30}$ G. Hermann, ${ }^{2}$ J. A. Hinton, ${ }^{2}$ W. Hofmann, ${ }^{2}$ C. Hoischen, ${ }^{28}$ T. L. Holch, ${ }^{31}$ M. Holler, ${ }^{32}$

D. Horns, ${ }^{25}$ D. Huber, ${ }^{32}$ H. Iwasaki, ${ }^{6}$ A. Jacholkowska, ${ }^{14} \S$ M. Jamrozy, ${ }^{33}$

D. Jankowsky, ${ }^{18}$ F. Jankowsky, ${ }^{29}$ L. Jouvin, ${ }^{26}$ I. Jung-Richardt, ${ }^{18}$ M. A. Kastendieck, ${ }^{25}$

K. Katarzyński, ${ }^{34}$ M. Katsuragawa, ${ }^{35}$ U. Katz ${ }^{18}$ D. Khangulyan, ${ }^{6}$ B. Khélifi, ${ }^{26}$ J. King, ${ }^{29}$ S. Klepser, ${ }^{12}$ W. Kluźniak, ${ }^{27}$ Nu. Komin, ${ }^{23}$ K. Kosack, ${ }^{16}$ M. Kraus, ${ }^{18}$ G. Lamanna, ${ }^{7}$ J. Lau, ${ }^{13}$ J. Lefaucheur, ${ }^{8}$ A. Lemière, ${ }^{26}$ M. Lemoine-Goumard, ${ }^{24}$ J.-P. Lenain, ${ }^{14}$ E. Leser, ${ }^{28}$ T. Lohse, ${ }^{31}$ R. López-Coto, ${ }^{2}$ M. Lorentz, ${ }^{16 \star}$ I. Lypova, ${ }^{12}$ D. Malyshev, ${ }^{20}$ V. Marandon, ${ }^{2}$ A. Marcowith, ${ }^{15}$ C. Mariaud, ${ }^{21}$ G. Martí-Devesa, ${ }^{32}$ R. Marx, ${ }^{2}$ G. Maurin, ${ }^{7}$ P. J. Meintjes, ${ }^{36}$ A. M. W. Mitchell, ${ }^{2}$ R. Moderski, ${ }^{27}$ M. Mohamed, ${ }^{29}$ L. Mohrmann, ${ }^{18}$ C. Moore,${ }^{37}$ E. Moulin, ${ }^{16}$ T. Murach, ${ }^{12}$

S. Nakashima, ${ }^{38}$ M. de Naurois, ${ }^{21}$ H. Ndiyavala, ${ }^{1}$ F. Niederwanger, ${ }^{32}$ J. Niemiec, ${ }^{22}$ L. Oakes, ${ }^{31}$ P. O'Brien, ${ }^{37}$ H. Odaka, ${ }^{39}$ S. Ohm, ${ }^{12}$ M. Ostrowski, ${ }^{33}$ I. Oya, ${ }^{12}$ M. Panter, ${ }^{2}$ R. D. Parsons, ${ }^{2}$ C. Perennes,${ }^{14}$ P.-O. Petrucci, ${ }^{30}$ B. Peyaud,${ }^{16}$ Q. Piel, ${ }^{7}$ S. Pita, ${ }^{26}$ V. Poireau, ${ }^{7}$ A. Priyana Noel, ${ }^{33}$ D. A. Prokhorov, ${ }^{23}$ H. Prokoph, ${ }^{12}$ G. Pühlhofer, ${ }^{20}$ M. Punch, ${ }^{10,26}$ A. Quirrenbach, ${ }^{29}$ S. Raab,${ }^{18}$ R. Rauth, ${ }^{32}$ A. Reimer, ${ }^{32}$ O. Reimer, ${ }^{32}$ M. Renaud, ${ }^{15}$ F. Rieger, ${ }^{2}$ L. Rinchiuso, ${ }^{16}$ C. Romoli, ${ }^{2}$ G. Rowell, ${ }^{13}$ B. Rudak, ${ }^{27}$

^E-mail: contact.hess@ @ess-experiment.eu

$\dagger$ Present address: Institut de Ciències del Cosmos (ICC UB), Universitat de Barcelona (IEEC-UB), Martí Franquès 1, E-08028 Barcelona, Spain. $\ddagger$ Funded by EU FP7 Marie Curie, grant agreement no. PIEF-GA-2012-332350.

$\S$ Deceased.

\ Present address: Physik Institut, Universität Zürich, Winterthurerstrasse 190, CH-8057 Zürich, Switzerland. 
E. Ruiz-Velasco, ${ }^{2}$ V. Sahakian, ${ }^{4,40}$ S. Saito, ${ }^{6}$ D. A. Sanchez, ${ }^{7}$ A. Santangelo, ${ }^{20}$ M. Sasaki, ${ }^{18}$ R. Schlickeiser, ${ }^{11}$ F. Schüssler, ${ }^{16}$ A. Schulz, ${ }^{12}$ H. Schutte, ${ }^{1}$ U. Schwanke, ${ }^{31}$ S. Schwemmer, ${ }^{29}$ M. Seglar-Arroyo, ${ }^{16}$ M. Senniappan, ${ }^{10}$ A. S. Seyffert, ${ }^{1}$ N. Shafi, ${ }^{23}$ I. Shilon, ${ }^{18}$ K. Shiningayamwe, ${ }^{9}$ R. Simoni,${ }^{17}$ A. Sinha,${ }^{26}$ H. Sol,${ }^{8}$ A. Specovius, ${ }^{18}$ M. Spir-Jacob,${ }^{26}$ Ł. Stawarz, ${ }^{33}$ R. Steenkamp, ${ }^{9}$ C. Stegmann, ${ }^{12,28}$ C. Steppa, ${ }^{28}$ T. Takahashi, ${ }^{35}$ J.-P. Tavernet, ${ }^{14}$ T. Tavernier, ${ }^{16}$ A. M. Taylor, ${ }^{12}$ R. Terrier,${ }^{26}$ D. Tiziani, ${ }^{18}$ M. Tluczykont,${ }^{25} \mathrm{C}$. Trichard,${ }^{21} \mathrm{M}$. Tsirou, ${ }^{15} \mathrm{~N}$. Tsuji, ${ }^{6} \mathrm{R}$. Tuffs, ${ }^{2}$ Y. Uchiyama, ${ }^{6}$ D. J. van der Walt, ${ }^{1}$ C. van Eldik, ${ }^{18}$ C. van Rensburg, ${ }^{1}$ B. van Soelen, ${ }^{36}$ G. Vasileiadis, ${ }^{15}$ J. Veh, ${ }^{18}$ C. Venter, ${ }^{1}$ P. Vincent,,${ }^{14}$ J. Vink, ${ }^{17}$ F. Voisin, ${ }^{13}$ H. J. Völk, ${ }^{2}$ T. Vuillaume, ${ }^{7}$ Z. Wadiasingh, ${ }^{1}$ S. J. Wagner, ${ }^{29}$ R. M. Wagner, ${ }^{41}$ R. White ${ }^{2}$ A. Wierzcholska, ${ }^{22}$ R. Yang, ${ }^{2}$ H. Yoneda, ${ }^{35}$ D. Zaborov, ${ }^{21}$ M. Zacharias, ${ }^{1}$ R. Zanin, ${ }^{2}$ A. A. Zdziarski, ${ }^{27}$ A. Zech, ${ }^{8}$ A. Ziegler, ${ }^{18}$ and J. Zorn ${ }^{2}$ N. Żywucka ${ }^{33}$

Affiliations are listed at the end of the paper

Accepted 2019 April 7. Received 2019 March 27; in original form 2019 January 25

\begin{abstract}
PKS 1830-211 is a known macrolensed quasar located at a redshift of $z=2.5$. Its highenergy gamma-ray emission has been detected with the Fermi-Large Area Telescope (LAT) instrument and evidence for lensing was obtained by several authors from its high-energy data. Observations of PKS 1830-211 were taken with the High Energy Stereoscopic System (H.E.S.S.) array of Imaging Atmospheric Cherenkov Telescopes in 2014 August, following a flare alert by the Fermi-LAT Collaboration. The H.E.S.S observations were aimed at detecting a gamma-ray flare delayed by $20-27 \mathrm{~d}$ from the alert flare, as expected from observations at other wavelengths. More than $12 \mathrm{~h}$ of good-quality data were taken with an analysis threshold of $\sim 67 \mathrm{GeV}$. The significance of a potential signal is computed as a function of the date and the average significance over the whole period. Data are compared to simultaneous observations by Fermi-LAT. No photon excess or significant signal is detected. An upper limit on PKS 1830211 flux above $67 \mathrm{GeV}$ is computed and compared to the extrapolation of the Fermi-LAT flare spectrum.
\end{abstract}

Key words: gravitational lensing: strong-diffuse radiation-gamma-rays: galaxies.

\section{THE PKS 1830-211 GRAVITATIONALLY LENSED QUASAR}

PKS 1830-211 is a high-redshift $(z=2.5$; Lidman et al. 1999) flat-spectrum radio quasar (FSRQ) that has been detected in all wavelengths from radio to high-energy gamma-rays. It is a known gravitationally lensed object with two compact images of the quasar nucleus visible in the radio (Jauncey et al. 1991) and optical (Meylan et al. 2005) passbands. The Einstein ring, well visible at radio frequencies, comes from the imaging of the quasar jet (Kochanek \& Narayan 1992). The quasar source is lensed by a foreground galaxy at $z=0.89$ (Wiklind \& Combes 1996). The angular size of the Einstein ring and separation of compact images is roughly 1 arcsec so that it cannot be resolved with high-energy instruments such as High Energy Stereoscopic System (H.E.S.S.; $50 \mathrm{GeV}-50 \mathrm{TeV}$ range) or Fermi-Large Area Telescope (LAT; $100 \mathrm{MeV}-100 \mathrm{GeV}$ range). PKS $1830-211$ is seen as a bright, highenergy source by the Fermi-LAT instrument and had several flaring periods during the decade of Fermi-LAT observations. PKS 1830211 is listed in the 1FHL (Ackermann et al. 2013) and the 3FHL (Ajello et al. 2017) catalogues with a photon index above $10 \mathrm{GeV}$ of $3.55 \pm 0.34$ that corresponds to the average 'low-state' spectrum. No significant curvature in the spectrum was detected. Photons up to $35 \mathrm{GeV}$, potentially detectable by H.E.S.S., have been observed by Fermi-LAT (Ajello et al. 2017). Observations of these very high energy photons and the measurement of the very high energy tail of the spectrum would give useful constraints on extragalactic background light (EBL) at redshift $z=2.5$.

Since the components of the lens cannot be resolved at high or very high energy, the evidence for lensing was searched indirectly on the observed light curve. Because of the different travel paths, the light curves of the two compact components of the lens have a relative time delay, measured in the radio (Lovell et al. 1998) and microwave (Wiklind \& Combes 2001) passbands, of $26 \pm 5 \mathrm{~d}$. Barnacka, Glicenstein \& Moudden (2011) have studied the first $3 \mathrm{yr}$ of the Fermi-LAT light curve with cepstral and autocorrelation 
methods. Evidence for a delay of $27.5 \pm 1.3 \mathrm{~d}$ was found with a $3 \sigma$ significance. The time delay between the compact images of PKS 1830-211 was also studied by the Fermi-LAT Collaboration (Abdo et al. 2015). They selected several flaring periods and calculated the autocorrelation function of the light curve. No significant peak was found. A possible peak of $\sim 20 \mathrm{~d}$ was found with a 1-d binning of the data, which could be attributed to the $\sim 20 \mathrm{~d}$ separation between two flaring events and perhaps to gravitational lensing. Barnacka et al. (2015) have argued that the time delay measured by high-energy instruments could be very different than the value measured by radio telescopes. The delay measured by Lovell et al. (1998) is obtained from the emission of the compact images. Since the jet of the PKS 1830-211 source is imaged close to the Einstein ring, the time difference between the initial burst and its lensed image can be much smaller if the source of high-energy emission is located inside the jet.

PKS 1830-211 is monitored by Fermi-LAT and its light curve is posted on the internet ${ }^{1}$ on a daily basis. H.E.S.S. observations of PKS 1830-211 were triggered by an alert posted by the Fermi-LAT team on 2014 August 2 (Krauss et al. 2014). The flare seen by the Fermi-LAT instrument started on July 27 and lasted $\sim 4 \mathrm{~d}$. The H.E.S.S. observations are described in Section 2 and data analysis in Section 3. The H.E.S.S. limits are compared to the Fermi-LAT signal in Section 4 and discussed in Section 5.

\section{H.E.S.S. OBSERVATIONS}

The very high energy (50 GeV-50 TeV range) gamma-ray observatory of the H.E.S.S. Collaboration consists of five Imaging Atmospheric Cherenkov Telescopes (IACTs) located in the Khomas Highland of Namibia $\left(23^{\circ} 16^{\prime} 18^{\prime \prime} \mathrm{S}, 16^{\circ} 30^{\prime} 1^{\prime \prime} \mathrm{E}\right), 1800 \mathrm{~m}$ above sea level. From 2004 January to 2012 October, the array was a fourtelescope instrument, with telescopes labelled CT1-4. Each of the telescopes, located at the corners of a square with a side length of $120 \mathrm{~m}$, has an effective mirror surface area of $107 \mathrm{~m}^{2}$, and is able to detect cosmic gamma-rays in the energy range 0.1-50 TeV. In 2012 October, a fifth telescope CT5, with an effective mirror surface area of $600 \mathrm{~m}^{2}$ and an improved camera (Bolmont et al. 2014), was installed at the centre of the original square, giving access to energies below $100 \mathrm{GeV}$ (H.E.S.S. Collaboration et al. 2017).

PKS 1830-211 was observed by the five telescopes of the H.E.S.S. IACT array between 2014 August 12 and August 26, to allow for the detection of delayed flares with time delays ranging from 20 to $27 \mathrm{~d}$. The observations were taken at an average zenith angle of $12^{\circ}$.

\section{DATA ANALYSES}

This paper is based on a sample of $12.4 \mathrm{~h}$ of high-quality data. Data selection cuts have been described in H.E.S.S. Collaboration et al. (2017). Data were next analysed with the Model analysis (de Naurois \& Rolland 2009) and cross-checked with the ImPACT analysis (Parsons \& Hinton 2014), the two methods giving compatible results. The two analyses use different calibration chains. With both reconstruction chains, data of CT5 were analysed either alone (Mono reconstruction) or combined with the CT1-4 data (Combined reconstruction). The Mono reconstruction has an energy threshold

${ }^{1}$ https://fermi.gsfc.nasa.gov/ssc/data/access/lat/msl_lc/source/PKS_1830-2 11

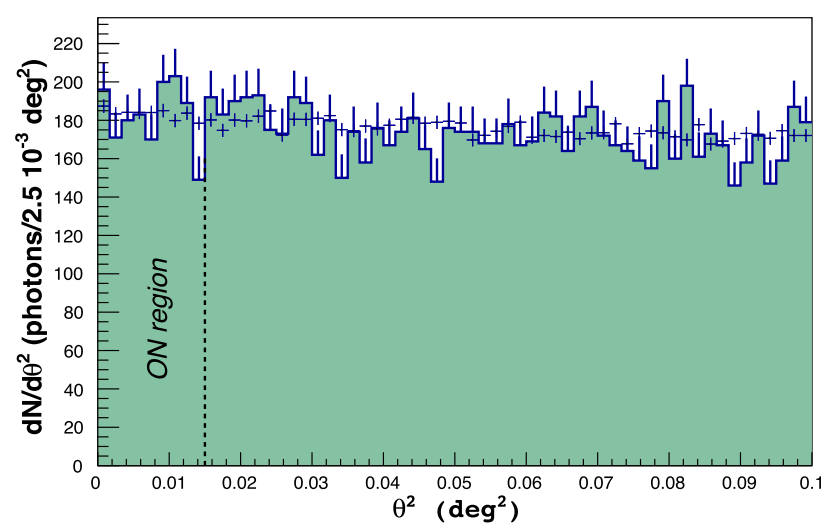

Figure 1. $\theta^{2}$ plot of PKS 1830-211 obtained with the Mono reconstruction. The background, shown by crosses, is estimated with the ring background method.

Table 1. Analysis results of observations of PKS $1830-211$ by H.E.S.S.

\begin{tabular}{lccc}
\hline Reconstruction & $N_{\mathrm{ON}}$ & $N_{\text {background }}$ & Significance $(\sigma)$ \\
\hline Mono & 1641 & 1649.2 & -0.2 \\
Combined & 935 & 954.4 & -0.6 \\
\hline
\end{tabular}

of $67 \mathrm{GeV}$. The Combined reconstruction has a higher threshold of $144 \mathrm{GeV}$, but a larger effective area.

A point source is searched at the location of PKS 1830-211. Fig. 1 shows the distribution of the squared angular distance $\theta^{2}$ of candidate photons from the target position. This distribution, obtained in the Mono analysis, is compared to the background from hadrons misidentified as photons. The background is calculated with the ring background method (Berge, Funk \& Hinton 2007), other methods giving similar results.

Table 1 summarizes the number of candidate photons in the signal region, the expected background, and the significance of the excess, calculated with $\mathrm{Li}$ and Ma formula 17 (Li \& Ma 1983). No significant excess of photons over background is seen by H.E.S.S. at the position of PKS 1830-211. A similar search using the Combined analysis also gives a negative result.

Because of the very soft spectrum measured by Fermi-LAT in the low state, PKS 1830-211 has a chance of being detectable by H.E.S.S. only during flares. The delayed flare lasts only less than about $4 \mathrm{~d}$, however, due to the uncertainties on the date of the flare, it could have happened at any time between August $17=$ MJD 56886 (time delay of $20 \mathrm{~d}$ ) and August 24 = MJD 56893 (radio time delay of $27 \mathrm{~d}$ ) as explained in Section 1. Fig. 2 shows the evolution over time of significance, binned by 28-min runs. No significant daily photon excess was detected during the H.E.S.S. observation period.

\section{FLUX UPPER LIMITS AND COMPARISON TO THE FERMI-LAT SPECTRA}

The non-detection by H.E.S.S. translates into 99 per cent confidence level (C.L.) upper limits on the average very high energy flux of PKS 1830-211 during H.E.S.S. observations. These upper limits are shown in Fig. 3. Red (respectively blue) arrows show the limits obtained from the Mono (respectively Combined) analysis and the corresponding solid lines show the effect of deabsorption using the EBL model of Gilmore et al. (2012). 


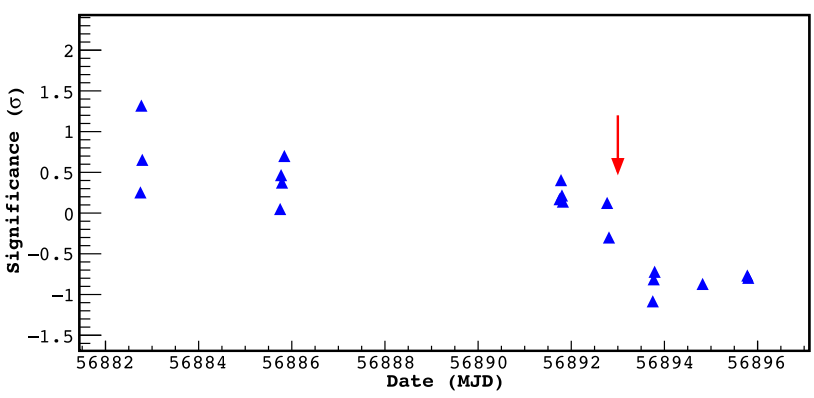

Figure 2. Significance of the H.E.S.S. signal versus date, obtained with the Mono analysis. The red arrow shows the expected date of the delayed flare for a lensing time delay of $27 \mathrm{~d}$.

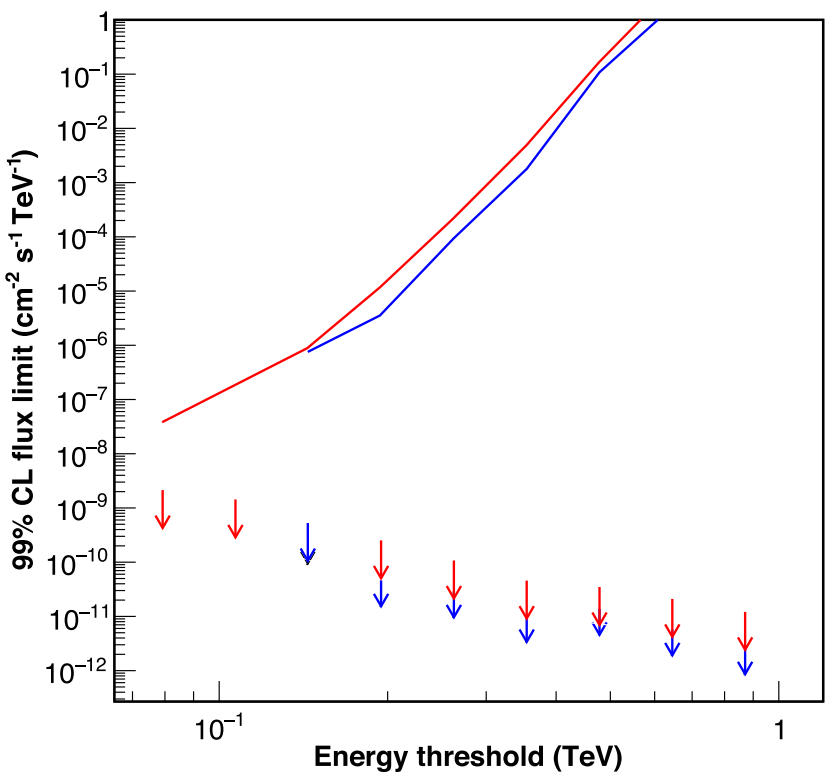

Figure 3. 99 per cent C.L. upper limits (arrows) on the PKS 1830-211 flux between $67 \mathrm{GeV}$ and $1 \mathrm{TeV}$ for the 2014 August H.E.S.S. observations. A constant photon index of -3 was assumed. The solid lines show the effect of EBL deabsorption, assuming the EBL model of Gilmore et al. (2012).

H.E.S.S. upper limits are compared to Fermi-LAT GeV spectra in Fig. 4. The Fermi-LAT observations have been analysed with Fermi Science Tools v10-r0p5 and Pass8 data, in the Enrico framework (Sanchez \& Deil 2013). The spectral data from 2014 July 26 to 30 (flare) are well described by a power-law spectrum with an index of $n_{\text {flare }}=-2.36 \pm 0.17$ for photon energies $>1 \mathrm{GeV}$. The relatively high low-energy cut was used to avoid contamination from the Galactic plane. The flare spectrum is much harder than the spectrum measured in the low state of PKS 1830-211, but $n_{\text {flare }}$ is compatible with the photon indices of previous flare spectra, as measured by Fermi-LAT (Abdo et al. 2015). The spectrum of PKS 1830-211 obtained from the Fermi-LAT observations within the H.E.S.S. observation window is well described by a power law with an index of $n_{\text {low }}=-2.97 \pm 0.44$ above $1 \mathrm{GeV}$. The value of $n_{\text {low }}$ is compatible with the value published in the 3 FHL catalogue.

A proper comparison between H.E.S.S. upper limits and the Fermi signal has to take into account the effect of the absorption of the flux of PKS 1830-211 by the EBL and the difference between the flare duration and H.E.S.S. exposure. Since no significant curvature of the spectrum was measured by the Fermi-LAT Collaboration, the unabsorbed spectrum was modelled by a power law. The effect of

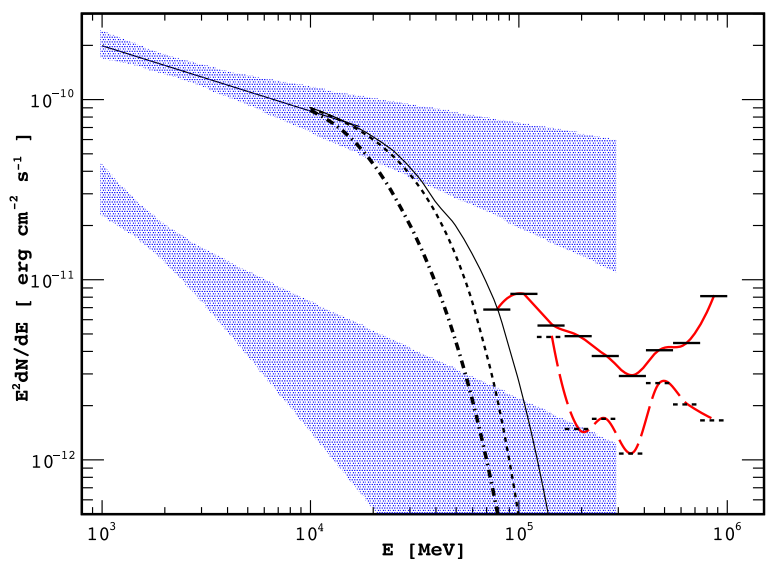

Figure 4. Comparison of H.E.S.S. flux 99 per cent C.L. upper limits (red solid line: Mono analysis; long dashed line: Combined analysis) to the measured spectra in the $\mathrm{GeV}$ region obtained with the Fermi-LAT data. The horizontal lines show the spectral resolution of the analyses. The lower blue butterfly is the $\mathrm{GeV}$ spectrum of PKS 1830-211 during H.E.S.S. observations. The upper blue butterfly is the corresponding spectrum during the 2014 July flare. The absorption of the July flare spectrum by EBL is calculated with models by Gilmore et al. (2012) (black dash-dotted line), Finke, Razzaque \& Dermer (2010) (dotted line), and Franceschini, Rodighiero \& Vaccari (2008) (black solid line).

light absorption by EBL from PKS 1830-211 has been estimated with the models of Gilmore et al. (2012) (black dash-dotted line), Finke et al. (2010) (dotted line), and Franceschini et al. (2008) (black solid line). Fig. 4 shows that there is a substantial difference between the predictions of these models for a source at redshift 2.5 such as PKS 1830-211. Note that EBL absorption could also be affected by the lens environment. Light from lensed active galactic nucleus (AGN) is expected to be more absorbed than average, due to the presence of galaxies along the line of sight. Indeed, absorption from the intervening galaxy has been detected by de Rosa et al. (2005) in the X-ray spectrum of PKS 1830-211. However, Barnacka, Böttcher \& Sushch (2014) and Böttcher \& Thiersen (2016) have argued that gravitational lensing could help gammarays from a distant source avoiding excess absorption. The FermiLAT flare spectrum from Fig. 4 is a 4-night average while the H.E.S.S. exposure amounts to 10 nights of data taking. The steady source upper limits from Fig. 3 are thus a factor of $\sim \sqrt{10 / 4}$ too constraining, which is corrected for in Fig. 4.

\section{CONCLUSION}

No significant delayed flare from PKS 1830-211 was detected by either H.E.S.S. or Fermi-LAT. The flare did not repeat or was too faint to be detected. Fig. 4 shows, however, that the detection of a strong flare would have been possible close to the Mono analysis energy threshold if the level of EBL absorption was at or below the absorption predicted by the model of Franceschini et al. (2008). Because of its lensed nature, observation of flaring event of PKS 1830-211 in the TeV passband could be useful to constrain EBL models at redshift as large as 2.5. The detection of the lensing time delay in future very high energy observations would help pinpoint the spatial origin of the high-energy emission (Barnacka et al. 2015). It would also permit more exotic applications such as constraining photon mass (Glicenstein 2017) or testing Lorentz invariance violation (Biesiada \& Piórkowska 2009). 


\section{ACKNOWLEDGEMENTS}

The support of the Namibian authorities and of the University of Namibia in facilitating the construction and operation of H.E.S.S. is gratefully acknowledged, as is the support by the German Ministry for Education and Research (BMBF), the Max Planck Society, the German Research Foundation (DFG), the Helmholtz Association, the Alexander von Humboldt Foundation, the French Ministry of Higher Education, Research and Innovation, the Centre National de la Recherche Scientifique (CNRS/IN2P3 and CNRS/INSU), the Commissariat à l' Energie Atomique et aux Energies Alternatives (CEA), the UK Science and Technology Facilities Council (STFC), the Knut and Alice Wallenberg Foundation, the National Science Centre, Poland grant no. 2016/22/M/ST9/00382, the South African Department of Science and Technology and National Research Foundation, the University of Namibia, the National Commission on Research, Science and Technology of Namibia (NCRST), the Austrian Federal Ministry of Education, Science and Research and the Austrian Science Fund (FWF), the Australian Research Council (ARC), the Japan Society for the Promotion of Science, and by the University of Amsterdam. We appreciate the excellent work of the technical support staff in Berlin, Zeuthen, Heidelberg, Palaiseau, Paris, Saclay, Tüebingen, and in Namibia in the construction and operation of the equipment. This work benefited from services provided by the H.E.S.S. Virtual Organisation, supported by the national resource providers of the EGI Federation.

\section{REFERENCES}

Abdo A. A. et al., 2015, ApJ, 799, 143

Ackermann M. et al., 2013, ApJS, 209, 34

Ajello M. et al., 2017, ApJS, 232, 18

Barnacka A., Glicenstein J.-F., Moudden Y., 2011, A\&A, 528, L3

Barnacka A., Böttcher M., Sushch I., 2014, ApJ, 790, 147

Barnacka A., Geller M. J., Dell'Antonio I. P., Benbow W., 2015, ApJ, 809, 100

Berge D., Funk S., Hinton J., 2007, A\&A, 466, 1219

Biesiada M., Piórkowska A., 2009, MNRAS, 396, 946

Bolmont J. et al., 2014, Nucl. Instrum. Methods Phys. Res. A, 761, 46

Böttcher M., Thiersen H., 2016, A\&A, 595, A14

de Naurois M., Rolland L., 2009, Astropart. Phys., 32, 231

de Rosa A. et al., 2005, A\&A, 438, 121

Finke J. D., Razzaque S., Dermer C. D., 2010, ApJ, 712, 238

Franceschini A., Rodighiero G., Vaccari M., 2008, A\&A, 487, 837

Gilmore R. C., Somerville R. S., Primack J. R., Domínguez A., 2012, MNRAS, 422, 3189

Glicenstein J.-F., 2017, ApJ, 850, 102

H. E. S. S. Collaboration et al., 2017, A\&A, 600, A89

Jauncey D. L. et al., 1991, Nature, 352, 132

Kochanek C. S., Narayan R., 1992, ApJ, 401, 461

Krauss F., Becerra J., Carpenter B., Ojha R., Buson S., 2014, Astron. Telegram, 6361

Li T.-P., Ma Y.-Q., 1983, ApJ, 272, 317

Lidman C., Courbin F., Meylan G., Broadhurst T., Frye B., Welch W. J. W., 1999, ApJ, 514, L57

Lovell J. E. J., Jauncey D. L., Reynolds J. E., Wieringa M. H., King E. A., Tzioumis A. K., McCulloch P. M., Edwards P. G., 1998, ApJ, 508, L51

Meylan G., Courbin F., Lidman C., Kneib J.-P., Tacconi-Garman L. E., 2005 , A\&A, 438, L37

Parsons R. D., Hinton J. A., 2014, Astropart. Phys., 56, 26

Sanchez D. A., Deil C., 2013, In Proceedings of the 33rd International Conference on Cosmic Rays (ICRC2013), Rio de Janeiro, Brazil, preprint (arXiv:1307.4534)

Wiklind T., Combes F., 1996, Nature, 379, 139
Wiklind T., Combes F., 2001, in Brainerd T. G., Kochanek C. S., eds, ASP Conf. Ser. Vol. 237, Gravitational Lensing: Recent Progress and Future Goals. Astron. Soc. Pac., San Francisco, p. 155

${ }^{1}$ Centre for Space Research, North-West University, Potchefstroom 2520, South Africa

${ }^{2}$ Max-Planck-Institut für Kernphysik, PO Box 103980, D-69029 Heidelberg, Germany

${ }^{3}$ Dublin Institute for Advanced Studies, 31 Fitzwilliam Place, Dublin 2, Ireland

${ }^{4}$ National Academy of Sciences of the Republic of Armenia, 24 Marshall Baghramian Avenue, 0019 Yerevan, Republic of Armenia

${ }^{5}$ Aix Marseille Université, CNRS/IN2P3, CPPM, 13009 Marseille, France ${ }^{6}$ Department of Physics, Rikkyo University, 3-34-1 Nishi-Ikebukuro, Toshima-ku, Tokyo 171-8501, Japan

${ }^{7}$ Laboratoire d'Annecy de Physique des Particules, Univ. Grenoble Alpes, Univ. Savoie Mont Blanc, CNRS, LAPP, F-74000 Annecy, France

${ }^{8}$ LUTH, Observatoire de Paris, PSL Research University, CNRS, Université Paris Diderot, 5 Place Jules Janssen, F-92190 Meudon, France

${ }^{9}$ Department of Physics, University of Namibia, Private Bag 13301, Windhoek, Namibia

${ }^{10}$ Department of Physics and Electrical Engineering, Linnaeus University, SE-351 95 Växjö, Sweden

${ }^{11}$ Institut für Theoretische Physik, Lehrstuhl IV: Weltraum und Astrophysik, Ruhr-Universität Bochum, D-44780 Bochum, Germany

${ }^{12}$ DESY, D-15738 Zeuthen, Germany

${ }^{13}$ School of Physical Sciences, University of Adelaide, Adelaide 5005, Australia

${ }^{14}$ Sorbonne Université, Université Paris Diderot, Sorbonne Paris Cité, CNRS/IN2P3, Laboratoire de Physique Nucléaire et de Hautes Energies, LPNHE, 4 Place Jussieu, F-75252 Paris, France

${ }^{15}$ Laboratoire Univers et Particules de Montpellier, Université Montpellier, CNRS/IN2P3, CC 72, Place Eugène Bataillon, F-34095 Montpellier Cedex

5, France

${ }^{16}$ IRFU, CEA, Université Paris-Saclay, F-91191 Gif-sur-Yvette, France

${ }^{17}$ GRAPPA, Anton Pannekoek Institute for Astronomy, University of Amsterdam, Science Park 904, NL-1098 XH Amsterdam, the Netherlands

${ }^{18}$ Friedrich-Alexander-Universität Erlangen-Nürnberg, Erlangen Centre for Astroparticle Physics, Erwin-Rommel-Str 1, D-91058 Erlangen, Germany

${ }^{19}$ Astronomical Observatory, The University of Warsaw, Al. Ujazdowskie 4, PL-00-478 Warsaw, Poland

${ }^{20}$ Institut für Astronomie und Astrophysik, Universität Tübingen, Sand 1, D-72076 Tübingen, Germany

${ }^{21}$ Laboratoire Leprince-Ringuet, Ecole Polytechnique, CNRS/IN2P3, F91128 Palaiseau, France

${ }^{22}$ Instytut Fizyki Jagdrowej PAN, ul. Radzikowskiego 152, PL-31-342 Kraków, Poland

${ }^{23}$ School of Physics, University of the Witwatersrand, 1 Jan Smuts Avenue, Braamfontein, Johannesburg 2050, South Africa

${ }^{24}$ Université Bordeaux, CNRS/IN2P3, Centre d'Études Nucléaires de Bordeaux Gradignan, F-33175 Gradignan, France

${ }^{25}$ Institut für Experimentalphysik, Universität Hamburg, Luruper Chaussee 149, D-22761 Hamburg, Germany

${ }^{26}$ APC, AstroParticule et Cosmologie, Université Paris Diderot, CNRS/IN2P3, CEA/Irfu, Observatoire de Paris, Sorbonne Paris Cité, 10 rue Alice Domon et Léonie Duquet, F-75205 Paris Cedex 13, France

${ }^{27}$ Nicolaus Copernicus Astronomical Center, Polish Academy of Sciences, ul. Bartycka 18, PL-00-716 Warsaw, Poland

${ }^{28}$ Institut für Physik und Astronomie, Universität Potsdam, Karl-LiebknechtStrasse 24/25, D-14476 Potsdam, Germany

${ }^{29}$ Landessternwarte, Universität Heidelberg, Königstuhl, D-69117 Heidelberg, Germany

${ }^{30}$ Univ. Grenoble Alpes, CNRS, IPAG, F-38000 Grenoble, France

${ }^{31}$ Institut für Physik, Humboldt-Universität zu Berlin, Newtonstr. 15, D12489 Berlin, Germany

${ }^{32}$ Institut für Astro- und Teilchenphysik, Leopold-Franzens-Universität Innsbruck, A-6020 Innsbruck, Austria 
${ }^{33}$ Obserwatorium Astronomiczne, Uniwersytet Jagielloński, ul. Orla 171, PL-30-244 Kraków, Poland

${ }^{34}$ Centre for Astronomy, Faculty of Physics, Astronomy and Informatics, Nicolaus Copernicus University, Grudziadzka 5, PL-87-100 Torun, Poland

${ }^{35}$ Kavli Institute for the Physics and Mathematics of the Universe (Kavli IPMU), The University of Tokyo Institutes for Advanced Study (UTIAS), The University of Tokyo, 5-1-5 Kashiwa-no-Ha, Kashiwa City, Chiba 277-8583, Japan

${ }^{36}$ Department of Physics, University of the Free State, PO Box 339, Bloemfontein 9300, South Africa

${ }^{37}$ Department of Physics and Astronomy, The University of Leicester, University Road, Leicester LE1 7RH, UK
${ }^{38}$ RIKEN, 2-1 Hirosawa, Wako, Saitama 351-0198, Japan

${ }^{39}$ Department of Physics, The University of Tokyo, 7-3-1 Hongo, Bunkyo-ku, Tokyo 113-0033, Japan

${ }^{40}$ Yerevan Physics Institute, 2 Alikhanian Brothers St, 375036 Yerevan, Armenia

${ }^{41}$ Oskar Klein Centre, Department of Physics, Stockholm University, Albanova University Center, SE-10691 Stockholm, Sweden

This paper has been typeset from a $\mathrm{T}_{\mathrm{E}} \mathrm{X} / \mathrm{L} \mathrm{T} \mathrm{E} \mathrm{X}$ file prepared by the author. 